\title{
A Comparison of Two Parameter Same Slope SEASONALITY AND HOLT-WINTERS EXPONENTIAL SMOOTHING MODELS
}

\author{
Hadis Bajric, Edin Kadric, Mugdim Pasic \& Izet Bijelonja
}
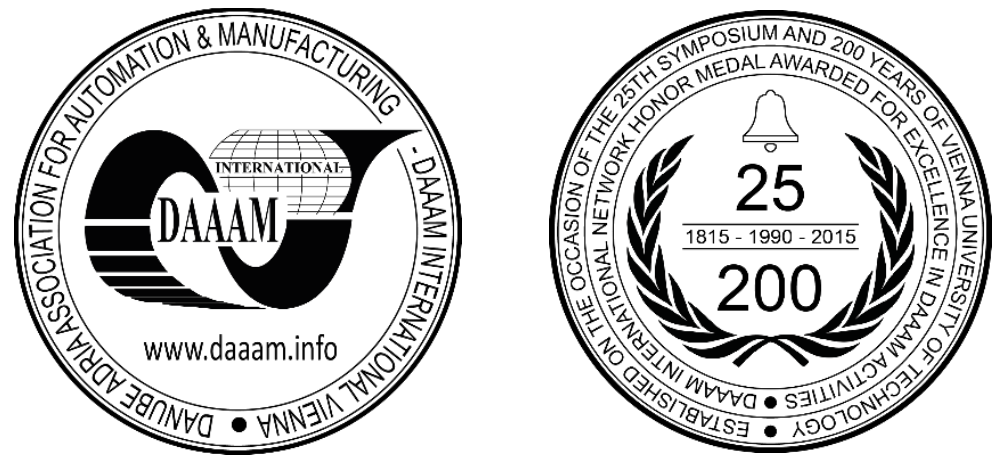

This Publication has to be referred as: Bajric, H[adis]; Kadric, E[din]; Pasic, M[ugdim]; Pasic, M[ugdim] \& Bijelonja, I[zet] (2017). A Comparison of Two Parameter Same Slope Seasonality and Holt-Winters Exponential Smoothing Models, Proceedings of the 28th DAAAM International Symposium, pp.0101-0108, B. Katalinic (Ed.), Published by DAAAM International, ISBN 978-3-902734-11-2, ISSN 1726-9679, Vienna, Austria

DOI: $10.2507 / 28$ th.daaam.proceedings.013

\begin{abstract}
In this paper two parameter Same Slope Seasonality forecasting model was developed. Analytical expressions for determining optimal parameter values of the developed two parameter Same Slope Seasonality forecasting model were developed too. Furthermore, performance of Two Parameter Same Slope Seasonality model was compared with performance of Holt-Winters exponential model as well as with performance of Same Slope Seasonality model. All tests were performed on M2-Competition time series. To determine the parameters of Holt-Winters exponential model, nonlinear mathematical programming was used. Performed tests proved that Two Parameter Same Slope Seasonality model is more successful than Holt-Winters exponential model and Same Slope Seasonality model. Expressions developed for determination parameters of two parameter Same Slope Seasonality forecasting model give parameter values identical to the values obtained by using Solver in MS Excel, set to solve nonlinear programming model, in a way that the problem is set up to search the parameter values which minimizes the mean squared error of the model.
\end{abstract}

Keywords: Holt-Winters model; Exponential smoothing; M2-Competition; Two Parameter Same Slope Seasonality forecasting model

\section{Introduction}

According to study of Green and Armstrong [1], regardless of the forecasting method increasing complexity leads to decrease in reliability. Authors further state that increase in complexity increases forecasting error for about $25 \%$. They suggest forecasters to use forecasting models they understand.

The reasons why Holt-Winters model (HW model) was taken for comparing to developed Two Parameter Same Slope Seasonality model (2pSSS model) and to Same Slope forecasting model (SS model) are:

- In time series analysis exponential smoothing methods are popular because they are straightforward and the whole forecasting procedure can happen automatically [2]. Yet it is often used in practice where it shows good performance.

- The HW model, also referred to as double exponential smoothing, is an extension of exponential smoothing designed for trended and seasonal time series. HW model is a widely used tool for forecasting business data that contain seasonality, changing trends and seasonal correlation [3]. 
- HW model is very robust and reliable, which in many studies shows close or better performances even than very complex ARIMA models [4, 5, 6, 7].

- HW model which is one of the most popular forecasting methods is utilized in cases where data show seasonality and/or trend. Probably the most important problem with HW model (apart from initialization) is setting the smoothing constants [8].

- Many companies use the HW model to produce short-term demand forecasts when their sales data contain a trend and a seasonal pattern. Fifty years old this year, the method is popular because it is simple, has low data-storage requirements, and is easily automated. It also has the advantage of being able to adapt to changes in trends and seasonal patterns in sales when they occur [9].

SS model and SSS model are distinguished by their simplicity and these models show better performances then some more complex exponential smoothing models. SS model, developed in [10], is one parameter model. SS model is of the same complexity as simple exponential smoothing model. In [10] testing and comparisons of SS model to fifteen exponential smoothing models are performed. Research results show that SS model is more efficient than simple exponential smoothing model. In [11] definition and test results of SSS model are presented. SSS model is one parameter model, which uses same logic as SS model, successfully models seasonality and with selection of adequate parameter value it successfully models trend component in available data. Research results show that developed model generates forecasts of the same reliability level as more complex exponential smoothing models.

In [12] analytical expression for estimate of optimal parameter value of SSS model are derived and presented. With this analytical expression SSS model has become even simpler for use. In the same research authors have compared performances of SSS model to HW model. Tests are performed on time series from M2-Competition [13] and SSS model has shown more reliable forecasts even SSS model is simpler than HW model. SSS model is one parameter model, while HW model is three parameter model. SSS model is defined by one expression and one parameter for level, trend and seasonality, while HW model uses three expressions and three parameters for every time series component. SSS model uses analytical expression for optimal parameter value estimation, while HW model requires use of nonlinear mathematical programming for estimation of optimal parameter values.

In this paper Two Parameter Same Slope Seasonality forecasting model (2pSSS model) is developed. This model should give better forecasts than SSS model because of its higher flexibility. In addition, analytical expressions for determination of both parameters are developed. Evaluation of developed model is performed on time series from M2Competition [13]. Performances of developed model are compared to performances of SSS model and HW model.

\section{Two Parameter Same Slope Seasonality model}

SS model is based on idea that time series will have the same gradient (slope) in the next time period like in previous. Model is defined by (1), whose settings are presented in [10]:

$$
\hat{X}_{t}(1)=X_{t}-\beta\left(X_{t-1}-X_{t}\right)
$$

Where is:

$X_{t} \quad$ - Observed value of the time series in time $t$,

$\hat{X}_{t}(1)$ - Predicted time series value one step forward,

$\beta \quad$ - Coefficient (parameter) of forecasting model.

SSS model is based on the same idea that change in time series between $t$ and $t+m$ will be the same to the change time series had in time period between $t-p$ and $t-p+m$. Graphical presentation of logic of SSS model is given on Fig. 1.

SSS model is also single parameter forecasting model defined by (2), whose settings are presented in [11]:

$$
\hat{X}_{t}(m)=X_{t}-\beta\left(X_{t-p}-X_{t-p+m}\right)
$$

Where is:

$X_{t} \quad$ - Observed value of the time series in time $t$,

$\hat{X}_{t}(m)$ - Predicted time series value $m$ step(s) forward, forecasted at time $t(m \leq p)$,

$m$ - Forecast horizon,

$p \quad$ - Number of seasons,

$\beta \quad$ - Coefficient (parameter) of forecasting models,

With respect to parameter $\beta$ following cases can be considered:

- $\beta<1$ - forecast smaller change rate in the period $t$ to $t+m$ than in the prior period, e.g. forecasts smaller change rate in the period $t$ to $t+m$ than in the period $t-p$ to $t-p+m$. 
- $\beta=1$ - forecast the same change rate in the period $t$ to $t+m$ as in the period $t-p$ to $t-p+m$.

- $\beta>1$ - forecast bigger change rate in the period $t$ to $t+m$ than in the period $t-p$ to $t-p+m$.

Considering (1) and (2) it can be concluded that in the case $p=1$, SSS model becomes SS model.

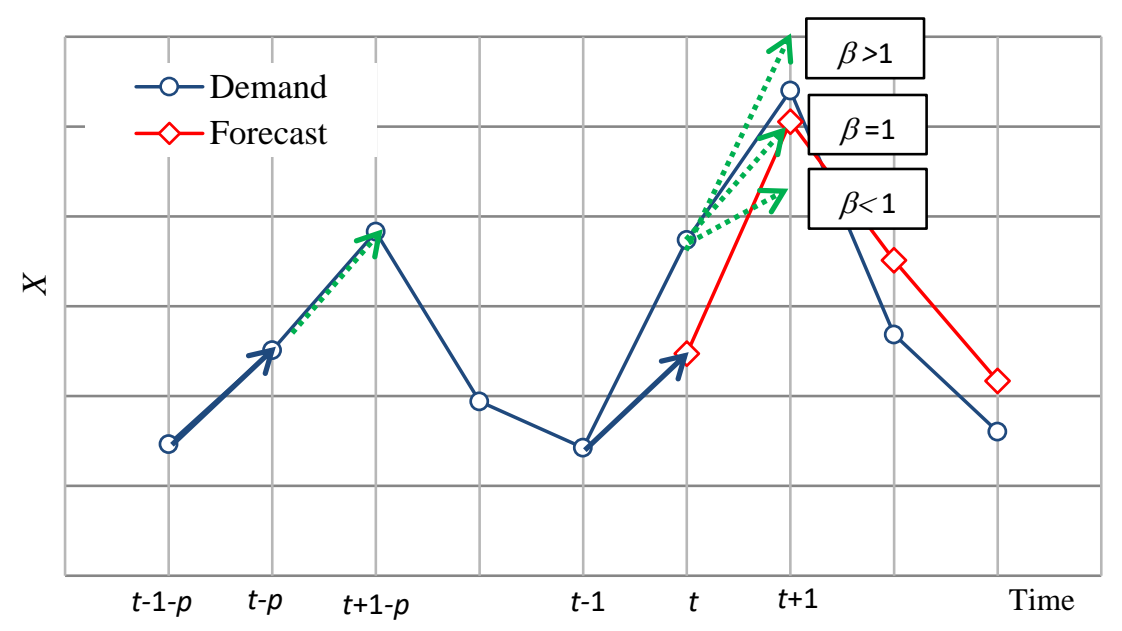

Fig. 1. Graphical presentation of logic of SSS model

Because of its mathematical simplicity SSS model allows finding analytical expression for determining optimal $\beta$ parameter. Analytical expression for calculating $\beta$ parameter is developed by [12]:

$$
\beta=\frac{\sum_{t=p+1}^{n+m-1}\left(X_{t}-X_{t+m}\right) \cdot\left(X_{t-p}-X_{t-p+m}\right)}{\sum_{t=p+1}^{n+m-1}\left(X_{t-p}-X_{t-p+m}\right)^{2}}
$$

Where $n$ is number of data in time-series.

The value of $\beta$ parameter calculated by (3) corresponds to the value determined using nonlinear mathematical programming, where standard deviation or MSE of model is minimized.

SSS model defined by expression (2) can be even more flexible if another coefficient is introduced. Adding another coefficient to SSS model, we define new model named Two Parameter SSS model by (4):

$$
\hat{X}_{t}(m)=\alpha X_{t}-\beta\left(X_{t-p}-X_{t-p+m}\right)
$$

Where is:

$X_{t} \quad$ - Observed value of the time series in time $t$,

$\widehat{X}_{t}(m)$ - Predicted time series value $m$ step(s) forward, forecasted at time $t(m \leq p)$,

$m$ - Forecast horizon,

$p \quad$ - Number of seasons,

$\alpha \quad$ - Coefficient (parameter) of forecasting models,

$\beta \quad$ - Coefficient (parameter) of forecasting models.

With respect to parameter $\alpha$ following cases can be considered:

- $\alpha<1$ - The last available data has impact on the next forecast in the lower rate than its value is.

- $\alpha=1$ - The last available data has impact on the next forecast in the same rate than its value is. In this case $2 \mathrm{pSSS}$ model become SSS model.

- $\alpha>1$ - The last available data has impact on the next forecast in the bigger rate than its value is.

Considering (1), (2) and (3) it can be concluded that in the case $\alpha=1,2$ pSSS model becomes SSS model. In the case $\alpha=1$ and $\beta=12$ pSSS model becomes SS model.

Graphical presentation of the logic of SSS model is given on Fig. 2 


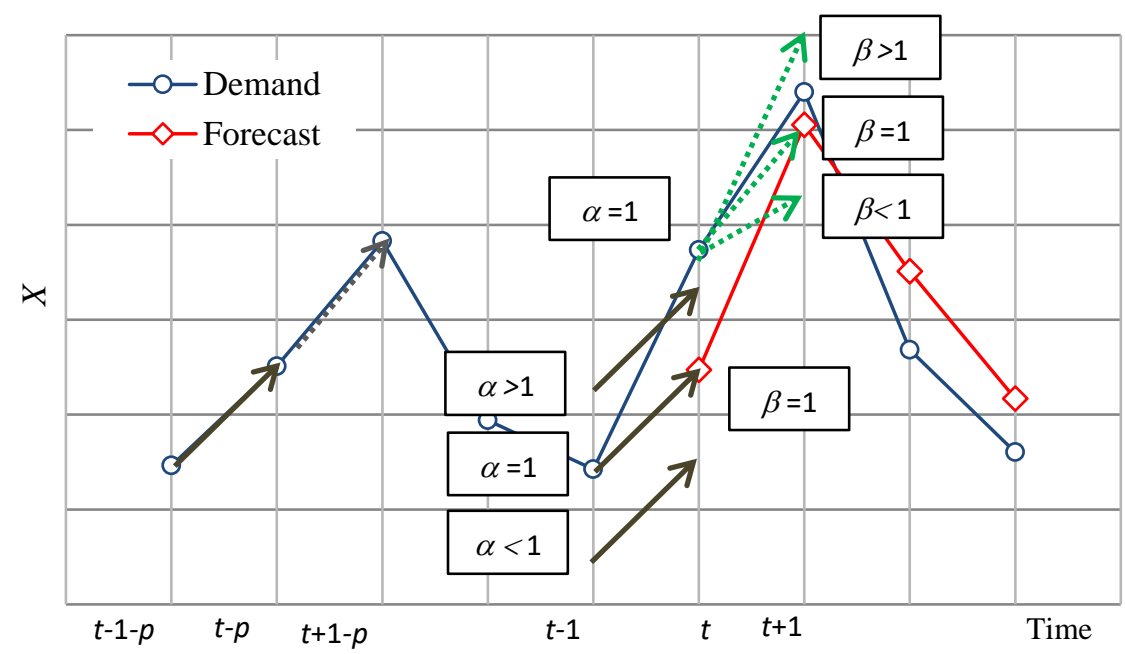

Fig. 2. Graphical presentation of logic of 2pSSS model

\section{Derivation of analytical expressions for determination of optimal parameter values of 2pSSS model}

Because of its mathematical simplicity, 2pSSS model allows derivation of expression for determining optimal $\alpha$ and $\beta$ parameters. It is necessary to formulate function of squared deviations between forecasted and true values, dependent on $\alpha$ and $\beta$ parameters, given by:

$$
f(\alpha, \beta)=\sum_{t=p+1}^{n+m-1}\left[X_{t+m}-\hat{X}_{t}(m)\right]^{2}
$$

Where $n$ is number of data in time-series.

Substituting (4) into (5) we get expression for sum of squared errors as the function of $\alpha$ and $\beta$ parameters:

$$
f(\alpha, \beta)=\sum_{t=p+1}^{n+m-1}\left[X_{t+m}-X_{t}-\beta\left(X_{t-p}-X_{t-p+m}\right)\right]^{2}
$$

In order to determine values for $\alpha$ and $\beta$ parameters, equation (6) is differentiated with respect to each coefficient. The first partial derivative of the function of squared errors $f(\alpha, \beta)$ with respect to $\alpha$ is given by:

$$
\frac{\partial f(\alpha, \beta)}{\partial \alpha}=\sum_{t=p+1}^{n+m-1} 2 \cdot\left[X_{t+m}-\alpha X_{t}+\beta\left(X_{t-p}-X_{t-p+m}\right)\right] \cdot\left(-X_{t}\right)
$$

The first partial derivative of the function of squared errors $f(\alpha, \beta)$ with respect to $\beta$ is given by:

$$
\frac{\partial f(\alpha, \beta)}{\partial \beta}=\sum_{t=p+1}^{n+m-1} 2 \cdot\left[X_{t+m}-\alpha X_{t}+\beta\left(X_{t-p}-X_{t-p+m}\right)\right] \cdot\left(X_{t-p}-X_{t-p+m}\right)
$$

Setting derivatives (7) and (8) equal to zero we get set of two linear equation with two unknowns ( $\alpha$ and $\beta$ ):

$$
\begin{aligned}
& \sum_{t=p+1}^{n+m-1}\left[X_{t+m} \cdot X_{t}-\alpha X_{t}^{2}+\beta\left(X_{t-p}-X_{t-p+m}\right) \cdot X_{t}\right]=0 \\
& \sum_{t=p+1}^{n+m-1} 2 \cdot\left[X_{t+m}-\alpha X_{t}+\beta\left(X_{t-p}-X_{t-p+m}\right)\right] \cdot\left(X_{t-p}-X_{t-p+m}\right)=0
\end{aligned}
$$


Solution of this two linear equation system provides optimal $\alpha$ and $\beta$ parameter values in explicit analytical form, defined by (11) and (12):

$$
\begin{aligned}
\alpha= & \frac{\sum_{t=p+1}^{n+m-1} X_{t+m} \cdot X_{t} \cdot \sum_{t=p+1}^{n+m-1}\left(X_{t-p}-X_{t-p+m}\right)^{2}}{\sum_{t=p+1}^{n+m-1} X_{t}^{2} \sum_{t=p+1}^{n+m-1}\left(X_{t-p}-X_{t-p+m}\right)^{2}-\left[\sum_{t=p+1}^{n+m-1} X_{t} \cdot\left(X_{t-p}-X_{t-p+m}\right)\right]^{2}}- \\
& -\frac{\sum_{t=p+1}^{n+m-1} X_{t+m} \cdot\left(X_{t-p}-X_{t-p+m}\right) \cdot \sum_{t=p+1}^{n+m-1}\left(X_{t-p}-X_{t-p+m}\right) \cdot X_{t}}{\sum_{t=p+1}^{n+m-1} X_{t}^{2} \sum_{t=p+1}^{n+m-1}\left(X_{t-p}-X_{t-p+m}\right)^{2}-\left[\sum_{t=p+1}^{n+m-1} X_{t} \cdot\left(X_{t-p}-X_{t-p+m}\right)\right]^{2}} \\
\beta= & \frac{\sum_{t=p+1}^{n+m-1}\left(\alpha X_{t}-X_{t+m}\right) \cdot\left(X_{t-p}-X_{t-p+m}\right)}{\sum_{t=p+1}^{n+m-1}\left(X_{t-p}-X_{t-p+m}\right)^{2}}
\end{aligned}
$$

The value of $\alpha$ and $\beta$ parameters calculated by (11) and (12) respectively corresponds to the value determined using nonlinear mathematical programming, where mean squared error or MSE of model is minimized.

\section{Research methodology}

Research methodology in this paper is same to the research methodology used in [12]. But here performances of 2pSSS model are compared with the performances of SSS model and with performances of three parameter HW model with additive trend and additive seasonal component, which is listed in standard list of exponential smoothing models [14]:

$$
\left.\begin{array}{c}
S_{t}=\alpha\left(X_{t}-I_{t-p}\right)+(1-\alpha)\left(S_{t-1}+T_{t-1}\right) \\
T_{t}=\gamma\left(S_{t}-S_{t-1}\right)+(1-\gamma) T_{t-1} \\
I_{t}=\delta\left(X_{t}-S_{t}\right)+(1-\delta) I_{t-p} \\
\hat{X}_{t}(m)=S_{t}+m T_{t}+I_{t-p+m}
\end{array}\right\}
$$

Where is:

$X_{t} \quad$ - Observed value of the time series in time $t$,

$\widehat{X}_{t}(m)$ - Predicted time series value $m \operatorname{step}(\mathrm{s})$ forward, forecasted at time $t(m \leq p)$,

$m$ - Forecast horizon,

$p \quad$ - Number of seasons,

$S_{t} \quad$ - Smoothed level of the series, computed after $X_{t}$ is observed,

$T_{t} \quad$ - Smoothed additive trend at the end of period $t$,

$I_{t} \quad$ - Smoothed seasonal index at the end of period $t$,

$\alpha \quad$ - Smoothing parameter for the level of the series,

$\gamma \quad$ - Smoothing parameter for the trend,

$\delta \quad$ - Smoothing parameter for seasonal indices.

Tests are performed on times series "M2CAll" from M2-Competition available in [15]. Last year data are used to estimate MAPE of the considered models, and all other data are used to estimate optimal values of forecasting models parameters.

Two Parameter SSS model parameters are determined using (11) and (12) for the case $m=1$. SSS model parameter is determined using (3) also for the case $m=1$. To determine HW model parameters, nonlinear mathematical programming is used. Nonlinear mathematical programming models are solved using Solver in Microsoft Excel application. The model standard deviation is used as the objective function of nonlinear mathematical programming model, also for the case $m=1$.

Forecasts are generated for time horizon of one year. After forecasts are generated, true time series values are used to estimate reliability measures. To compare model performances MAPE is used as a measure of model reliability. Forecasts are generated using only quantitative data and without any available qualitative information.

Tests are performed on 28 of 29 time series from M2-Competition. Time series INTERSAL haven't been used in tests, because its value in the last year is 0 , which prevents calculating MAPE value. Among 28 analyzed, 22 were monthly and 6 quarterly time series. 


\section{Results}

The developed expressions for determination $\alpha$ and $\beta$ parameters in 2pSSS model, for each of the tested time series, give identical values for both $\alpha$ and $\beta$ parameters to the values obtained by using Solver in MS Excel. The value of $\alpha$ and $\beta$ parameters calculated by (11) and (12) respectively corresponds to the value determined using nonlinear mathematical programming, where mean squared error or MSE of model is minimized.

MAPE values for both models and all analyzed time series are given in Table 1. It can be seen from Table 1. that 2pSSS model has better performances for 15 time series, while HW model has for 13. Also it can be seen that $2 \mathrm{pSSS}$ model has better performances for 16 time series, while HW model has for 12.

Average MAPE values for 2pSSS, SSS and HW are 25,56\%, 24,25\%, 24,03\% respectively. If three time series (PANTER, PHARMA11 and TRADUSAL), for which both models have high uncertainty levels, are omitted from analysis, then average MAPE values for 2pSSS, SSS and HW are 11,64\%, 11,80\% and 12,41\%, respectively.

\begin{tabular}{|l|c|c|c|}
\hline Series & HW model & SSS model & 2pSSS model \\
\hline PANTER & $284,54 \%$ & $259,65 \%$ & $253,75 \%$ \\
\hline CHEETAH & $18,39 \%$ & $17,43 \%$ & $14,66 \%$ \\
\hline LION & $29,45 \%$ & $37,15 \%$ & $36,31 \%$ \\
\hline BIGCAT & $13,50 \%$ & $15,34 \%$ & $15,01 \%$ \\
\hline ANIMAL & $14,47 \%$ & $16,94 \%$ & $16,14 \%$ \\
\hline CARINDS & $13,03 \%$ & $12,85 \%$ & $12,53 \%$ \\
\hline REALGNP & $0,45 \%$ & $7,06 \%$ & $2,11 \%$ \\
\hline IPDGNP & $0,17 \%$ & $0,79 \%$ & $0,29 \%$ \\
\hline IPDIMP & $2,55 \%$ & $0,97 \%$ & $1,46 \%$ \\
\hline FIXINV & $4,60 \%$ & $4,43 \%$ & $4,73 \%$ \\
\hline COMPIND & $1,44 \%$ & $0,35 \%$ & $0,35 \%$ \\
\hline BUSINV & $10,24 \%$ & $7,48 \%$ & $13,22 \%$ \\
\hline OEMUSAL & $18,00 \%$ & $10,32 \%$ & $10,86 \%$ \\
\hline TRADUSAL & $36,32 \%$ & $58,16 \%$ & $44,33 \%$ \\
\hline FLMARKT & $22,54 \%$ & $8,26 \%$ & $8,97 \%$ \\
\hline RESDVSAL & $10,87 \%$ & $7,79 \%$ & $7,33 \%$ \\
\hline INDSHIPF & $10,49 \%$ & $9,30 \%$ & $9,27 \%$ \\
\hline PHARMA11 & $41,73 \%$ & $102,92 \%$ & $90,02 \%$ \\
\hline PHARMA22 & $30,04 \%$ & $21,50 \%$ & $23,21 \%$ \\
\hline PHARMA33 & $28,48 \%$ & $23,59 \%$ & $24,10 \%$ \\
\hline METOGEN & $24,25 \%$ & $23,64 \%$ & $22,18 \%$ \\
\hline CARDERS & $23,23 \%$ & $22,40 \%$ & $21,09 \%$ \\
\hline NEWRX & $3,66 \%$ & $2,97 \%$ & $2,98 \%$ \\
\hline REFILLS & $4,32 \%$ & $2,73 \%$ & $2,73 \%$ \\
\hline UNCPAPEUR & $7,08 \%$ & $18,37 \%$ & $17,92 \%$ \\
\hline CPAPEUR & $8,59 \%$ & $10,21 \%$ & $10,20 \%$ \\
\hline UNCPAPUSA & $5,34 \%$ & $6,99 \%$ & $7,16 \%$ \\
\hline CPAPUSA & $5,18 \%$ & $6,17 \%$ & $6,13 \%$ \\
\hline
\end{tabular}

Table 1. Comparative display of MAPE values for all analyzed time-series

It can be concluded, based on test results shown in Table 1 that 2 pSSS forecasting model shows better performances than more complex HW forecasting model on respectable number of time series. The same can be concluded for $2 \mathrm{pSSS}$ with regard to SSS model. Important advantage of 2pSSS in relation to HW model is existence of analytical expressions 
for direct calculation of optimal $\alpha$ and $\beta$ parameter values. For estimation of optimal parameter values of HW model it is necessary to use nonlinear mathematical programming and appropriate solver applications. Importance of developed model is even bigger if it is known that HW model is very robust and reliable, which in many studies shows close or better performances even than very complex ARIMA models [4, 5, 6, 7].

Tests show that 2pSSS model is less sensitive on current observation in time series which is main disadvantage of SSS model. So, 2pSSS model obtains better results than SSS model, in almost all time series with outliers. For example, see MAPE values for time series PANTER, LION, TRADUSAL, PHARMA 11, in Table 1.

Even accuracies of 2pSSS model and SSS model forecasts significantly depend on current observation in time series, and it does not mean that models are not usable in such situations. We consider that forecasting of such time series requires inclusion of some qualitative information. For comparison, HW model which usually has more stabile forecasts, when forecasting such time series can result with quite poor forecasts. One of examples of such time series is PANTER, shown on Fig. 3. For PANTER time series, MAPE values for SSS model, 2pSSS model and HW model are 259,65\%, 253,75\%, $284,54 \%$, respectively. From the MAPE values it can be seen that HW performed with highest value, eg. with poorest forecasts.

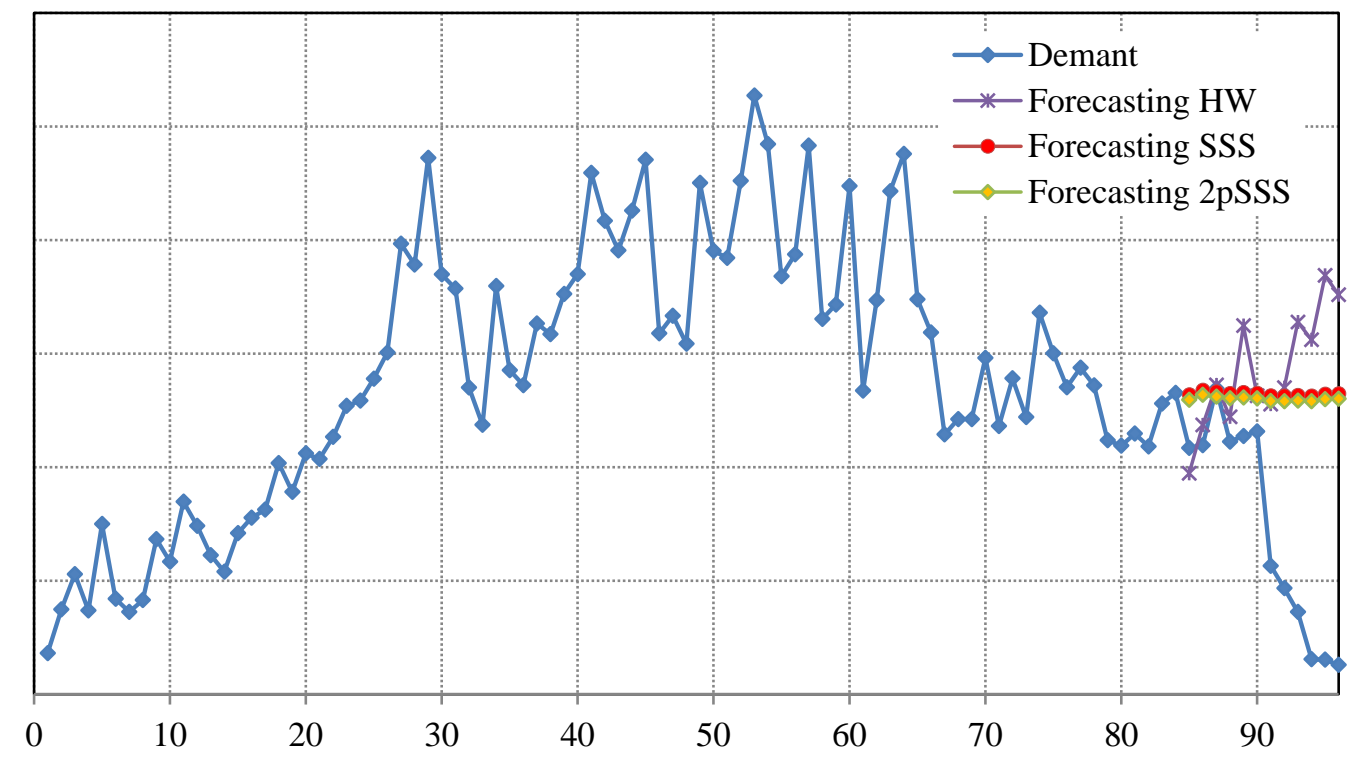

Fig. 3. PANTER Time series

\section{Conclusion}

The new 2pSSS model, which was developed in this paper, is a very simple and easy to use model. The research results have shown that new 2pSSS model has great potential which worth and which need to be examined in different situations. Two Parameter SSS model shows better results than the famous HW model in the tests performed on 28 M2-Competition time series. Two Parameter SSS model has less MAPE value on 15, while HW model has on 13 time series. Also 2pSSS model shows better results than SSS model. Two Parameter SSS model has less MAPE value on 16, while SSS model has on 12 time series.

In this paper, the analytical expressions for determining optimal parameter values of the developed Two Parameter Same Slope Seasonality forecasting model were developed too. The idea for developing these analytical expressions is simple and it has same logic as a least squares method. Determine the value of parameters which give the best fit forecast. These analytical expressions, for each of the tested time series, give $\alpha$ and $\beta$ values identical to the values obtained by using Solver in MS Excel, set to solve nonlinear programming model, in a way that the problem is set up to search the $\alpha$ and $\beta$ parameters which minimizes the mean squared error of the model.

Comparative advantage of 2pSSS model to HW model especially is important when considering costs of parameter determination. Parameters of 2 pSSS model are simply determined using analytical expressions, while parameters determination in case of HW model requires solving nonlinear mathematical programming model. Besides this obvious advantage, 2pSSS model is easier to understand and implement than HW model, and also it requires less computational operations and efforts in forecast generations. Forecasts generated by 2 pSSS model are of the same or better reliability levels as forecasts generated by more complex HW model. 
Two Parameter SSS model performs well in forecasting time series with dominant seasonal, as well as trend component. Unreliable forecasts by 2pSSS model can be expected in case of non-standard observations in the time series, because 2pSSS model forecasts are very dependent on current observation for which forecasting value is generated. However, tests have shown that 2 pSSS model is less sensitive on current observation in time series which is main disadvantage of SSS model. Finally, developed 2pSSS model performs better than SSS model. Advantage of 2pSSS model is especially emphasized in time series with outliers and in time series with short term interventions (quantum change of time series level).

It can be concluded that developed 2pSSS model, as well as SSS model, could be more effective if, for different seasons, different $\beta$ parameter values are used. Use of this approach would not make models more complex, but it requires estimation of number of different $\beta$ parameter values equivalent to number of different seasons. Even with additional calculations, 2pSSS model and SSS model would be simpler than HW model. Besides, the future research should be focused on testing developed model on other cases in different fields.

\section{References}

[1] Green, C. K. \& Armstrong, J. S. (2015). Simple versus complex forecasting: The evidence. Journal of Business Research, Volume 68, Issue 8, pp. 1678-1685

[2] Crevits, R. \& Croux, C. (2016). Forecasting with Robust Exponential Smoothing with Damped Trend and Seasonal Components, Working Paper, Available from:

https://www.researchgate.net/profile/Ruben_Crevits/publication/310638788_Forecasting_with_Robust_Exponentia 1_Smoothing_with_Damped_Trend_and_Seasonal_Components/links/58347e5808ae102f073957f1.pdf Accessed: 2017-07-05, DOI: 10.13140/RG.2.2.11791.18080

[3] Gelper, S.; Fried, R. \& Croux, C. (2010). Robust Forecasting with Exponential and Holt-Winters Smoothing. Journal of Forecasting, 29, 285-300.

[4] Makridakis, S. \& Hibon, M. (2000). The M3-Competition: results, conclusions and implications. International Journal of Forecasting, 16 (2000) 451-476.

[5] Puthran, D.; Shivaprasad, H. C.; Keerthesh Kumar, K. S. \& Manjunath, M. (2014). Comparing SARIMA and HoltWinters' forecasting accuracy with respect to Indian motorcycle industry, Transactions on Engineering and Sciences 2 (5) (2014)

[6] Tanvir, K. (2011). Identifying an Appropriate Forecasting Model for Forecasting Total Import of Bangladesh, International Journal of Trade, Economics and Finance, 2 (3) (2011)

[7] Walters, Q. C. (2008). Investigating the use of Holt-Winters time series model for forecasting population at the state and sub-state levels. Demographics and Workforce Section, Weldon Cooper Center for Public Service, University of Virginia, 2008.

[8] Williams, T. M. (1987). Adaptive Holt-Winters Forecasting, Journal of the Operational Research Society 38 (6) pp. 553-560.

[9] Goodwin, P., 2010. The Holt-Winters approach to exponential smoothing: 50 years old and going strong. Foresight: The International Journal of Applied Forecasting 19, 30-33.

[10]Pašić, M.; Bijelonja, I.; Šunje, A. \& Bajrić, H. (2007). Same Slope Forecasting Method, Proceedings of the 18th International DAAAM Symposium, B. Katalinic, (Ed.), (2007) 547-548.

[11]Bajrić, H.; Bijelonja, I. \& Pašić, M. (2009). A Comparison of Same Slope Seasonality and Exponential Smoothing Forecasting Models, Proceedings of the 20th International DAAAM Symposium, B. Katalinic, (Ed.), (2009) 13431344.

[12] Bajric, H.; Kadric, E. \& Pasic, M. (2016). A Comparison of Same Slope Seasonality and Holt-Winters Smoothing Forecasting Models, Proceedings of the 26th DAAAM International Symposium, pp.0062-0068, B. Katalinic (Ed.), Published by DAAAM International, ISBN 978-3-902734-07-5, ISSN 1726-9679, Vienna, Austria, DOI:10.2507/26th.daaam.proceedings.009

[13] Makridakis, S.; Chatfield, C.; Hibon, M.; Lawrence, M.; Mills, T.; Ord, K. \& Simmons, L. F. (1993). The M2Competition: A real-time judgmentally based forecasting study, International Journal of Forecasting, 9 (1993) 5-22.

[14]E. S. Gardner, Exponential smoothing: The state of the art - Part II, International Journal of Forecasting, 22 (4) (2006) 637-666.

[15] IIF, International Institute of Forecasters, M2-Competition, Available from: http://forecasters.org/resources/timeseries-data/m2-competition/ Accessed: 2015-05-30 